\title{
LOS TATUAJES COMO MÉTODO DE AUTOLESIÓN PARA DISMINUIR EL DOLOR PSÍQUICO EN PERSONAS CON TRASTORNO LÍMITE DE LA PERSONALIDAD
}

\author{
VANESSA PERICÁS ÁNGEL'1, ORIOL SÀNCHEZ MARQUÈS² Y TERESA SOLÉ BLASI ${ }^{3}$ \\ ${ }^{1}$ Enfermera de la Sala de Agudos de Psiquiatría. Hospital de la Santa Creu i Sant Pau. Barcelona. \\ 2Enfermero. CAS Sant Pau. Hospital de la Santa Creu i Sant Pau. Barcelona. \\ ${ }^{3}$ Enfermera del Hospital de Día de Trastornos de la Conducta Alimentaria. \\ Hospital de la Santa Creu i Sant Pau. Barcelona.
}

\section{RESUMEN}

Introducción: estudios previos muestran como muchas prácticas autolesivas físicas contribuyen a disminuir el dolor psíquico y la ansiedad, a la vez que exponen que el trastorno límite de la personalidad (TLP) es la orientación diagnóstica que más realiza esta práctica. El objetivo de este estudio es entender si los tatuajes son concebidos como un método autolesivo por parte de los pacientes con TLP para poder orientar nuestra práctica asistencial a la hora de realizar intervenciones de prevención de las autolesiones y de la impulsividad, intervenciones de prevención de los tatuajes como método autolesivo e intervenciones dirigidas a ofrecer otras formas de control y canalización de la impulsividad y de la expresión de la ansiedad.

Material y método: estudio observacional, descriptivo y transversal donde se incluyó a 36 pacientes diagnosticados de TLP portadores de, al menos, un tatuaje. Todos ellos fueron atendidos en alguna de las unidades de psiquiatría del Hospital de la Santa Creu i Sant Pau de Barcelona entre enero y junio de 2016. A través de un cuestionario ad hoc diseñado específicamente para este estudio, se evaluó de forma individualizada cada tatuaje, en cuanto a: i) si los participantes consideraban que sus tatuajes habían sido realizados como método de autolesión y ii) las características de cada tatuaje (motivo de realización, lugar, temática, impulsividad). Además, todos los participantes completaron el Cuestionario de Impulsividad de Barratt (BIS-11).

Resultados: participaron 36 pacientes diagnosticados de TLP (33 mujeres y 3 hombres), con una media de edad de 35 años (desviación estándar [DE]: 7,945) y una media de tatuajes de 3,89 (DE: 2,816). Se analizaron 140 tatuajes, de los cuales, 83 se realizaron por placer; 33, por control; 29, por dolor psíquico; 26, por rabia; y 17, por ansiedad. La localización más frecuente fue en las extremidades superiores $(n=68)$ y la temática más recurrente fueron símbolos y logos $(n=51)$. De los 140 tatuajes, 76 fueron realizados como método autolesivo según los participantes, siendo las principales motivaciones de realización el placer $(n=29)$, el dolor psíquico $(n=28)$ y la rabia $(n=25)$. De los tatuajes realizados como método autolesivo, 
30 estaban relacionados con malos tratos/abusos. No se presentaron diferencias entre el número de tatuajes como método autolesivo y el grado de impulsividad.

Discusión: los tatuajes fueron realizados como método autolesivo en más de la mitad de los casos entre los pacientes con TLP, siendo las principales motivaciones el dolor psíquico, la rabia y la ansiedad.

Palabras clave: tatuaje, autolesión, trastorno límite de la personalidad, TLP, ansiedad, dolor psíquico.

\section{INTRODUCCIÓN}

El trastorno límite de la personalidad (TLP) es, según la quinta edición del Manual diagnóstico y estadístico de los trastornos mentales (DSM-5), un trastorno que se caracteriza primariamente por la inestabilidad emocional, el pensamiento extremadamente polarizado y dicotómico, una notable impulsividad y relaciones interpersonales caóticas ${ }^{1}$. El perfil global del trastorno incluye típicamente una inestabilidad generalizada del estado del ánimo, de la autoimagen, de la conducta y del sentido de la identidad. Se inicia al principio de la edad adulta y algunos de los criterios diagnósticos del TLP son, por ejemplo, presentar un patrón de relaciones interpersonales inestables e intensas caracterizadas por la alternancia entre los extremos de idealización y devaluación, ira inapropiada e intensa o dificultades para controlarla, intentos o amenazas suicidas recurrentes o comportamientos de automutilación ${ }^{1}$.

Se calcula que la prevalencia del TLP es de, aproximadamente, un $2 \%$ de la población en general y que este trastorno se diagnostica en el $10 \%$ de los pacientes de centros ambulatorios de salud mental y cerca del $20 \%$ de los pacientes psiquiátricos ingresados. Por lo que respecta a la distribución por sexos, predomina en las mujeres en una proporción de 3:1. Entre el 60 y el $80 \%$ de los pacientes con TLP han presentado comportamientos autolesivos, lo cual ele-

Correspondencia: Vanessa Pericás Ángel Correo electrónico: vpericas@santpau.cat va el riesgo de suicidio. Las tentativas de suicidio son la causa más frecuente de consulta a urgencias de psiquiatría y de ingreso hospitalario en este tipo de pacientes. Entre el 8 y el $10 \%$ acaban consumando el suicidio, mayoritariamente, hombres de entre 30 y 35 años ${ }^{1}$.

\section{Estado actual del tema}

En el ámbito de la salud mental, Swami et al., del Departamento de Psicología de la Universidad de Westminster, concluían que, de los 440 encuestados, el $19,8 \%$ y el $15,2 \%$ tenía piercings y tatuajes, respectivamente. Además, reflejaba que las mujeres eran más propensas a tener piercings en el cuerpo que los hombres, pero no había diferencias significativas de sexo en los tatuajes ${ }^{2}$. Aunque no existen datos disponibles sobre la asociación de la autolesión a los tatuajes, Carroll et al. concluyeron que los participantes con tatuajes y/o piercings eran más propensos a participar en comportamientos de riesgo y a hacerlo en grados de participación más elevada (entendiendo estos comportamientos como alteraciones alimentarias, consumo de drogas, actividad sexual o suicidio). La violencia se asoció a los hombres que tenían tatuajes y a las mujeres que tenían perforaciones en el cuerpo, y el suicidio se asoció a mujeres que tenían tatuajes y menores de edad con tatuajes y piercings ${ }^{3}$. Stirn et al. evaluaron la asociación de la modificación corporal en la población alemana mediante tatuajes a las condiciones percibidas 
negativamente de la vida, a la reducción de la integración social y al aumento de comportamientos de búsqueda de sensaciones ${ }^{4}$. Stieger et al. evaluaron los tatuajes en 540 personas en la Europa Central: un $22 \%$ de los encuestados tenían algún tatuaje realizado, y estos presentaban una puntuación más alta por lo que respecta a la extroversión, la búsqueda de experiencia, la necesidad de singularidad y la búsqueda de emociones ${ }^{5}$.

Asimismo, existen numerosos estudios dentro del ámbito de la salud mental que han incidido en las formas clásicas de autolesión en personas diagnosticadas de TLP, como puede ser el caso del estudio de Nock et al., en el que se analiza la autolesión entendida como un acto deliberado de causarse daño a uno mismo mediante cortes, quemaduras, mutilaciones u otros métodos traumáticos, excluyendo las sobreingestas medicamentosas o envenenamientos con finalidad autolítica. En este estudio, se ofrecen dos resultados relevantes interesantes para nuestro trabajo: que dos tercios de los adolescentes hospitalizados en psiquiatría son mujeres con una historia reciente de autolesiones, de las cuales, la mitad cumplen criterios diagnósticos para ser diagnosticadas de TLP; y que hay una elevada tasa de intentos de suicidio en la muestra de adolescentes que han participado recientemente de prácticas autolesivas ${ }^{6}$.

\section{Justificación}

Los datos de los estudios muestran como muchas prácticas autolesivas físicas (cortes, heridas y quemaduras básicamente) contribuyen a disminuir el dolor psíquico y la ansiedad, a la vez que exponen que el TLP es la orientación diagnóstica que más realiza esta práctica. Siendo conocedores de esta información, y debido al incremento de la realización de tatuajes en pacientes con TLP, entender si los tatuajes son concebidos como un método autolesivo por parte de los pacientes con TLP puede ser relevante en la práctica asistencial a la hora de realizar intervenciones de prevención de las autolesiones y de la impulsividad, intervenciones de prevención de los tatuajes como método autolesivo e intervenciones dirigidas a ofrecer otras formas de control y canalización de la impulsividad y de la expresión de la ansiedad.

\section{Objetivos}

\section{Objetivo general}

Determinar la prevalencia de tatuajes en pacientes con TLP que son utilizados como método autolesivo.

\section{Objetivos específicos}

- Analizar la correlación entre la motivación de la autolesión con cortes y/o quemaduras y los tatuajes.

- Examinar la temática y localización más frecuente de los tatuajes en personas con TLP.

- Determinar los principales factores de riesgo que se asocian a la realización de tatuajes como método autolesivo en personas con TLP.

- Analizar la asociación entre la impulsividad como rasgo de la personalidad y la realización de tatuajes como método autolesivo.

\section{MATERIAL Y MÉTODO}

Estudio observacional, analítico y transversal. La población de estudio fueron los pacientes adultos con diagnóstico clínico de TLP y portadores de tatuajes que acudieron a consultas externas del servicio de salud mental, a urgencias de psiquiatría o que ingresaron en la unidad de hospitalización de psiquiatría de nuestro hospital entre enero y junio de 2016, que cumplieron con los criterios de selección y que aceptaron participar en el estudio.

\section{Criterios de inclusión:}

- Pacientes adultos con tatuajes y diagnóstico clínico de TLP según los criterios del DSM-IV-TR, eje II. 
- Pacientes que lleven asociados diagnósticos de trastorno de dependencia de sustancias y/o trastornos de la conducta alimentaria, según los criterios del DSM-IV-TR, eje I.

\section{Criterios de exclusión:}

- Pacientes con enfermedades mentales graves (DSM-IV-TR, eje I).

- Déficit cognitivo.

La unidad de estudio son los tatuajes.

Las variables analizadas son:

- Variable dependiente: prevalencia de tatuajes como método autolesivo.

- Definición conceptual: definiremos los tatuajes como métodos autolesivos según lo descrito en el DSM-5, en el capítulo de afecciones que necesitan más estudio, donde se define la autolesión no suicida como una "conducta por medio de la cual el individuo se aplica intencionadamente lesiones en la superficie corporal del tipo que suelen producir sangrado, hematoma 0 dolor, con la expectativa de que la lesión solo conlleve dolor físico leve o moderado (por ejemplo: cortes, quemaduras, golpes)» ${ }^{1}$.

- Definición operativa: para este estudio, consideraremos los tatuajes como métodos autolesivos cuando los motivos de realización de los tatuajes coincidan con cortes (Sí/NO) y quemaduras (SÍ/NO) (métodos autolesivos según la definición del DSM-5). Los motivos evaluados en este estudio para la realización de tatuajes serán: ansiedad, dolor psíquico, rabia, placer y control.

Para el estudio evaluaremos la prevalencia de tatuajes como método autolesivo (número total de tatuajes como método autolesivo × 100/número total de tatuajes evaluados).

\section{- Variables independientes:}

- Temática de los tatuajes.

- Localización de los tatuajes.
- Factores personales:

1. Edad (años).

2. Sexo (mujer/hombre).

3. Impulsividad: se evaluará mediante la Escala de Impulsividad de Barratt ${ }^{7}$, que es una escala autoaplicada de 30 ítems, agrupados en tres subescalas de impulsividad: cognitiva, motora e impulsividad no planeada. Cada ítem consta de cuatro opciones de respuesta. La puntuación en cada subescala se obtiene sumando las puntuaciones parciales obtenidas en cada uno de sus ítems, mientras que la puntuación total se obtiene sumando las puntuaciones obtenidas en todos los ítems. Dado que no existe ningún punto de corte, para este estudio, utilizaremos la mediana de la distribución y se tratará como una variable cuantitativa.

4. Procesos vitales traumáticos (procesos de la vida que han influenciado, determinado e inspirado la realización de los tatuajes: ninguno/muerte de un familiar, abusos sexuales, maltratos, otros).

Para determinar la muestra necesaria para el estudio, se decidió realizar un muestreo no probabilístico y consecutivo, en el que se incluyó a todos los pacientes que cumplían con los criterios de selección y quisieron participar en el estudio. Asumiendo una prevalencia del $15 \%$, una precisión del $5 \%$ y sin pérdidas estimadas, fue suficiente una muestra aleatoria de 137 tatuajes, partiendo de una población de 150 pacientes por año con TLP y una mediana de tres tatuajes por paciente.

Durante el período de estudio, cualquier miembro del equipo investigador realizó el cribado para la selección de pacientes elegibles y solicitó el consentimiento informado a los pacientes. A los pacientes que aceptaron participar en el estudio, se les realizó una entrevista en la que el investigador recogió datos personales, laborales, clínicos y del tratamiento médico. Además, se pasó un cuestionario autoconfec- 
cionado específicamente para este estudio y un cuestionario validado de impulsividad. Como fuente de datos, se utilizaron las historias clínicas de los pacientes.

Inicialmente, se procedió a la descripción de todas las variables; para las variables categóricas (tatuaje como método autolesivo, temática de los tatuajes, localización, sexo, procesos traumáticos), se facilitó el porcentaje con el número de casos y, para las variables cuantitativas (edad, impulsividad), se calculó el valor medio, con su desviación estándar (DE). Los datos de la prevalencia de tatuajes en pacientes con TLP realizados como método autolesivo, así como la temática, la localización más recurrente y los factores más prevalentes que motiven su realización (ansiedad, dolor psíquico, rabia, placer y control) se presentaron con su intervalo de confianza (IC del $95 \%$ ). La asociación entre cada uno de estos factores y el tatuaje como método autolesivo se evaluó por separado mediante la prueba de la chi al cuadrado $\left(\chi^{2}\right)$. Para analizar los principales factores de riesgo personales que se asociaron a la realización de tatuajes como método autolesivo (variable categórica), se utilizó la prueba de la $t$ de Student para variables cuantitativas (edad, grado de impulsividad). Para las variables categóricas (sexo, procesos vitales traumáticos), se utilizó la prueba de la $\chi^{2}$.

Para analizar los datos, se diseñó una base de datos en formato de Excel y, posteriormente, el análisis estadístico se realizó con el programa SPSS (versión 21.0), fijándose la probabilidad de cometer un error de tipo I en un $5 \%(\alpha=0,05)$ con una aproximación bilateral.

En cuanto a las limitaciones del estudio, se tuvo en cuenta que su realización podía presentar algún sesgo de información relacionado con el propio diseño del estudio. Al tratarse de un estudio con un cuestionario de preguntas abiertas, podían darse omisiones y traspasos incorrectos de la información a los cuestionarios autoconfeccionados. Con la finalidad de controlar este tipo de sesgos, se utilizó el cuestionario estructurado de impulsividad y, para la com- plementación del cuestionario de autolesiones y tatuajes, únicamente participaron los tres investigadores.

Después de la realización del protocolo de estudio, este fue presentado al Comité Ético de Investigación Clínica del Hospital de la Santa Creu i Sant Pau, que aceptó el estudio y permitió la realización del cribado y la obtención de datos de la forma anteriormente citada, a la vez que garantizaba la realización de control, auditoría, revisión e inspección del ensayo por las autoridades competentes.

\section{RESULTADOS}

Han participado en el estudio 36 personas, con edades comprendidas entre los 18 y los 52 años, siendo la media de edad de los participantes de 35 años (DE: 7,945). De los 36 participantes, 33 eran mujeres y 3 eran hombres. La media de tatuajes de los participantes es de 3,89 tatuajes, siendo la mediana y la moda 3 tatuajes. La media resultante del test de Barratt para los encuestados ha sido de 66,94 puntos, siendo la mediana 67,00 puntos. La moda se ha encontrado para los valores 62 y 78 , donde ambos han contado con tres casos cada uno.

Han sido analizados 140 tatuajes, de los cuales, 83 se realizaron por placer; 33, por control; 29, por dolor psíquico; 26, por rabia; y 17, por ansiedad, teniendo en cuenta que los participantes podían escoger más de una opción de respuesta. De los 140 tatuajes, 68 se localizaron en las extremidades superiores; 32, en las extremidades inferiores; 14 , en la espalda; 8 , en el abdomen; 6 , en el tórax; 5 , en la cadera y glúteos/ingles; y 2 , en la cara. La distribución más recurrente de las temáticas del total de tatuajes es la siguiente: símbolos/logos $(n=51)$, animales ( $n=19)$, nombres $(n=18)$, palabras/frases $(n=18)$, flores/plantas $(n=15)$, objetos $(n=9)$, personas/personajes $(n=7)$ y cenefas $(n=3)$.

De los 140 tatuajes, en el 36,4\%, no había ningún hecho relevante que hubiera propiciado su realización; el 32,1 \% escogieron la opción de otros hechos 
relevantes como desencadenante para la realización del tatuaje; el 21,4\% fueron por malos tratos/abusos; el 5,7\%, por muerte de un familiar; y el 4,3\%, por el nacimiento de un hijo/a. De los tatuajes realizados por otros hechos relevantes ( $n=45)$, destaca que el $27,5 \%$ se realizaron por libertad/equilibrio; el $25 \%$, por amor; y el $10 \%$, por familia; así como que un $7,5 \%$ estaba relacionado con enfermedad, y un $2,5 \%$, con ideación o intentos suicidas.

Del total de tatuajes ( $n=140)$, un $54,30 \%(n=76)$ fueron realizados como método autolesivo según los participantes, siendo las principales motivaciones de realización el placer $(n=29)$, el dolor psíquico $(n=$ $28)$, la rabia $(n=25)$, el control $(n=20)$ y la ansiedad $(n=14)$, como se muestra en la figura 1.

De los tatuajes realizados como autolesión $(n=76)$, las localizaciones se distribuyeron de la siguiente manera: extremidades superiores $(n=40)$, extremidades inferiores ( $n=14$ ), espalda ( $n=9)$, glúteos/ ingles $(n=4)$, abdomen $(n=4)$, cadera $(n=3)$, tórax $(n=1)$ y cara $(n=1)$, como se muestra en la figura 2 .
Por lo que se refiere a las temáticas de los tatuajes entendidos como autolesivos, las más recurrentes fueron símbolos/logos ( $n=23)$, nombres $(n=12)$, objetos ( $n=9)$, flores/plantas ( $n=9)$, letras/palabras $(n=9)$, animales ( $n=9)$, personas/personajes $(n=4)$ y cenefas $(n=1)$ (fig. 3 ).

En cuanto a la relación entre los tatuajes como autolesión ( $n=76$ ) y los hechos relevantes, el $39,47 \%(n=30)$ está relacionado con malos tratos/ abusos, mientras que un 27,63\% $(n=21)$ no está relacionado con ningún hecho relevante, el 19,75\% $(n=15)$ está relacionado con otros hechos relevantes, el $7,89 \%(n=6)$ con la muerte de un familiar y el $5,26 \%(n=4)$ con el nacimiento de un hijo/a. Dentro de los tatuajes vinculados con otros hechos relevantes ( $n=15)$, los participantes expusieron que estos se asociaban a búsqueda de libertad/equilibrio $(n=5)$, amor $(n=3)$, odio $(n=3)$, enfermedad $(n=2)$ e ideación/tentativa autolítica $(n=2)$ (fig. 4).

Un $35,53 \%(n=27)$ de los tatuajes realizados como autolesión pertenecían a pacientes procedentes de la sala de hospitalización; un 34,21 \% ( $n=26)$,

Figura 1. Motivaciones de los tatuajes como método de autolesión.

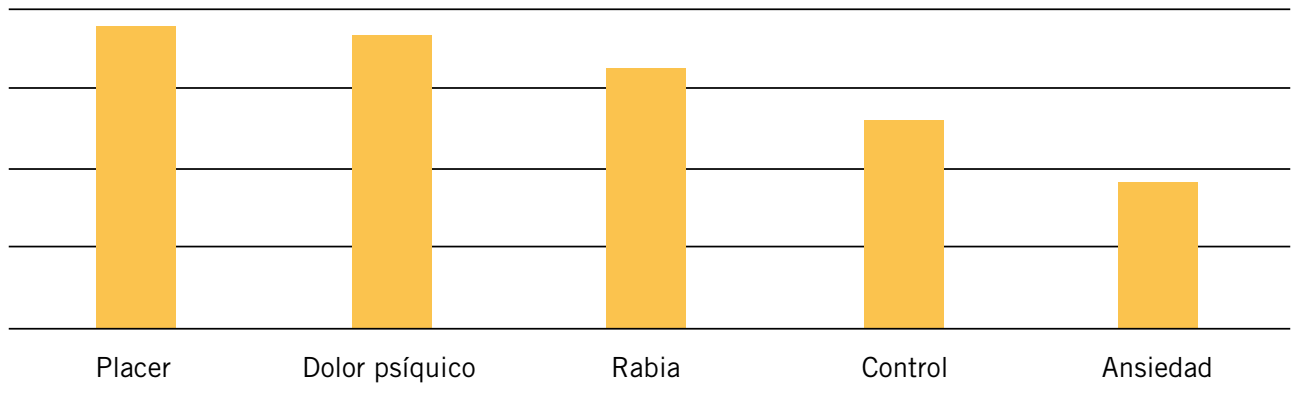




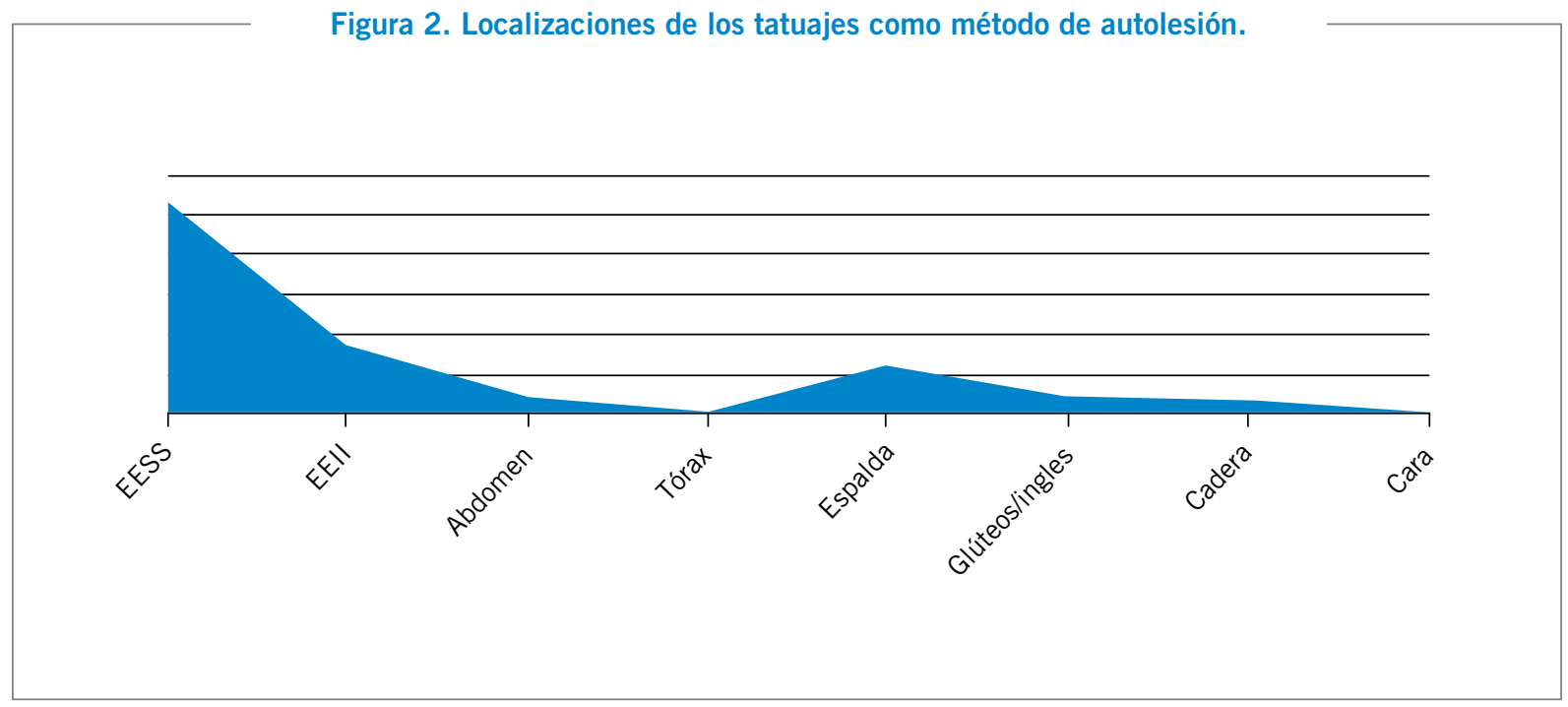

EEII: extremidades inferiores; EESS: extremidades superiores.

Figura 3. Temáticas de los tatuajes como método de autolesión.

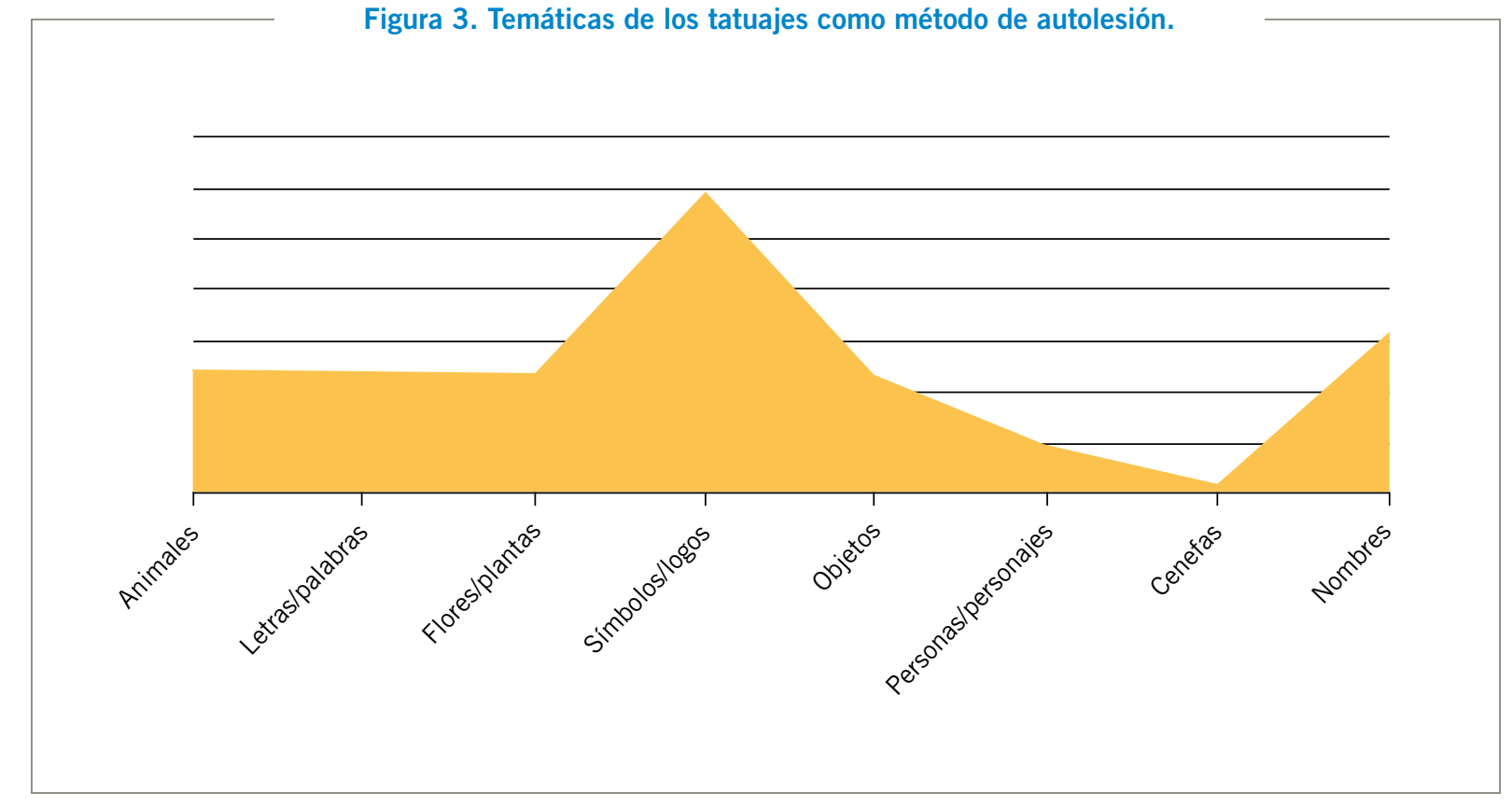

de consultas externas de trastornos de la conducta alimentaria; un $18,42 \%$ ( $n=14)$, de urgencias de psiquiatría; y un $11,84 \%$ ( $n=9$ ), de consultas externas de toxicomanías.
La media de impulsividad según el test de Barratt en pacientes portadores de tatuajes entendidos como una forma de autolesión es de 71,80 puntos (DE: $11,856)$, lo cual muestra que hay una tendencia a 


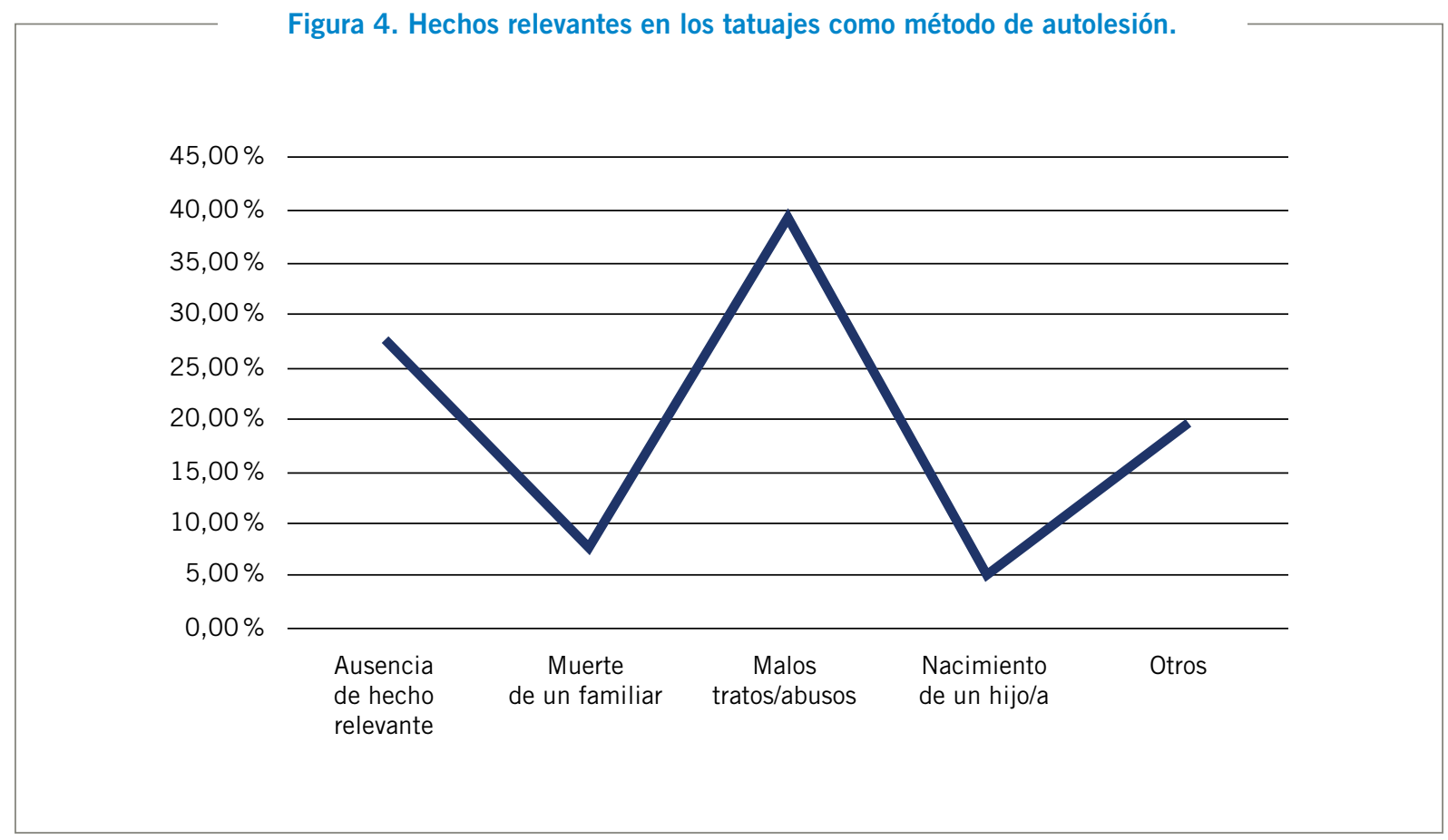

que, a mayor impulsividad, existe una mayor probabilidad de realizarse tatuajes como métodos autolesivos.

\section{DISCUSIÓN}

Los tatuajes fueron realizados como método autolesivo en más de la mitad de los casos entre los pacientes con TLP, siendo las principales motivaciones de realización por placer, dolor psíquico y rabia. La media de impulsividad según el test de Barratt de los participantes que eran portadores de tatuajes como método autolesivo era superior a la media de todos los participantes. Este hecho podía entreverse en estudios previos en los que las personas portadoras de tatuajes tenían un perfil caracterizado por un carácter extrovertido, deseoso de realizar actividades que les proporcionaran nuevas sensaciones y experiencias y con una elevada propensión a participar en actividades de riesgo.
Tanto para los tatuajes realizados como método autolesivo como para los que no eran considerados autolesión, las localizaciones más frecuentes eran las extremidades superiores e inferiores, mientras que sí existían diferencias en el grupo de localizaciones menos frecuentes: para los tatuajes autolesivos, estas eran la cara y el tórax y, para el conjunto de tatuajes en general, eran la cara, la cadera y los glúteos/ingles. Así pues, las extremidades, tanto superiores como inferiores, eran las localizaciones más frecuentes, pero es interesante ver que todos los tatuajes realizados en la cara fueron considerados como método de autolesión, lo cual podría explicarse como una manera extrema de canalización en la expresión de sentimientos negativos asociados a su situación (rabia o dolor psíquico) de manera pública, aunque, en el momento de la entrevista, se arrepentían de la impulsividad al realizárselo, verbalizando arrepentimiento por haberlo hecho.

Por lo que se refiere a las temáticas, para el conjunto general de tatuajes, las más realizadas son 
símbolos/logos, animales, nombres y palabras/frases, mientras que las menos frecuentes son personas/ personajes y cenefas. En el caso de los tatuajes como autolesión, existe una mayor igualdad en cuanto a la temática realizada, aunque destaca como más frecuente los símbolos/logos, lo que resulta interesante por la asociación existente entre la simbología plasmada en los tatuajes y las diferentes motivaciones de realización de estos, volviendo a ser una vía de plasmación impulsiva del estado en el que se encontraban los participantes.

En cuanto a los hechos relevantes vinculados a la realización del tatuaje, destaca que, en un elevado porcentaje de tatuajes entendidos como método autolesivo, estos hayan sido los malos tratos/abusos, mientras que el resto de valores son similares en comparación con el total de los tatuajes analizados. En la bibliografía relacionada con el TLP, resulta característico el hecho de que son personas con patrones de relaciones interpersonales inestables e intensas caracterizadas por la alternancia entre los extremos de idealización y devaluación, ira inapropiada e intensa o dificultades para controlarla, así como que muchas veces se encuentran siendo partícipes de relaciones tóxicas y relaciones en las que los maltratos y abusos constituyen un pilar característico, siendo necesario destacar que lo anteriormente citado ocurre en todo tipo de relaciones de su vida: con familia, parejas, amigos, etc.

Para finalizar, se resaltará la significativa diferencia existente entre la valoración subjetiva que presentan los pacientes al no entender el tatuaje como método autolesivo y la valoración objetiva mediante el cuaderno de recogida de datos, en que más de la mitad de los tatuajes analizados son entendidos como método de autolesión. Esta diferencia podría deberse al hecho de que, en el imaginario colectivo, no se valora el tatuaje como un acto tan impulsivo como un corte, una quemadura o una autolesión. No obstante, si entendemos el concepto de «autolesión» como una conducta por medio de la cual el individuo se aplica intencionadamente lesiones en la superficie corporal del tipo que suelen producir sangrado, hematoma o dolor, con la expectativa de que la lesión solo conlleve dolor físico leve o moderado, disminuyendo el dolor psíquico y la ansiedad, en este caso, el tatuaje sí sería una tipología más de autolesión, tal y como se ha valorado en el presente estudio.

Sería interesante ampliar este estudio desde un punto de vista cualitativo para complementar nuestros resultados y configurar una realidad hasta ahora desconocida, como es la valoración de los tatuajes como una nueva forma de autolesión, que nos permita conocer más y mejor a los usuarios a los que atendemos y, de esta forma, individualizar la atención enfermera hacia ellos.

\section{BIBLIOGRAFÍA}

1. American Psychiatric Association. Diagnostic and statistical manual of mental disorders. 5. ${ }^{\mathrm{a}}$ ed. Washington D.C.: American Psychiatric Association; 2013.

2. Swami V, Pietschnig J, Bertl B, Nader IW, Stieger S, Voracek M. Personality differences between tattooed and non-tattooed individuals. Psychol Rep. 2012;111(1): 97-106.

3. Carroll ST, Riffenburgh RH, Roberts TA, Myhre EB. Tattoos and body piercings as indicators of adolescent risk-taking behaviors. Pediatrics. 2002;109(6):1021-7.

4. Stirn A, Hinz A, Brähler E. Prevalence of tattooing and body piercing in Germany and perception of health, mental disorders, and sensation seeking among tattooed and body-pierced individuals. J Psychosom Res. 2006;60(5):531-4.

5. Stieger S, Pietschnig J, Kastner CK, Voracek M, Swami V. Prevalence and acceptance of tattoos and piercings: a survey of young adults from the southern Germanspeaking area of Central Europe. Percept Mot Skills. 2010;110(3 Pt 2):1065-74.

6. Nock MK, Joiner TE Jr, Gordon KH, Lloyd-Richardson E, Prinstein MJ. Non-suicidal self-injury among adolescents: diagnostic correlates and relation to suicide attempts. Psychiatry Res. 2006;144(1):65-72.

7. Oquendo MA, Baca-García E, Graver R, Morales M, Montalván V, Mann JJ. Spanish adaptation of the Barratt Impulsiveness Scale (BIS-11). Eur J Psychiatry. 2011; 15(3):147-55. 